\title{
CLOSED SUBMODULES IN THE MODULE OF ENTIRE FUNCTIONS OF EXPONENTIAL TYPE AND POLYNOMIAL GROWTH ON THE REAL AXIS
}

\author{
N.F. ABUZYAROVA
}

\begin{abstract}
In the work we consider a topological module $\mathcal{P}$ of entire functions, which is the isomorphic image under the Fourier-Laplace transform of Schwarz space $\mathcal{E}^{\prime}$ of distributions compactly supported in a finite or infinite interval $(a ; b) \subset \mathbb{R}$. We study some properties of closed submodules in module $\mathcal{P}$ related with local description problem. We also study issues on duality between closed submodules in $\mathcal{P}$ and subspaces in the space $\mathcal{E}=C^{\infty}(a ; b)$ invariant w.r.t. the differentiation.
\end{abstract}

Keywords:entire functions, Fourier-Laplace transform, local description of submodules, invariant subspaces, spectral synthesis, finitely generated submodules.

Mathematics Subject Classification: 30D15, 30H99, 42A38, 47E05

\section{INTRODUCTION}

For a finite or infinite interval $(a ; b)$ on the real axis we consider a sequence of segments exhausting this interval: $\left[a_{1} ; b_{1}\right] \Subset\left[a_{2} ; b_{2}\right] \Subset \ldots$ Let $P_{k}$ be the Banach space formed by all entire function $\varphi$ with finite norm

$$
\|\varphi\|_{k}=\sup _{z \in \mathbb{C}} \frac{|\varphi(z)|}{(1+|z|)^{k} \exp \left(b_{k} y^{+}-a_{k} y^{-}\right)}, \quad y^{ \pm}=\max \{0, \pm y\}, \quad z=x+\mathrm{i} y,
$$

$\mathcal{P}$ be the inductive limit of sequence $\left\{P_{k}\right\}$. Each of the embedding $P_{k} \subset P_{k+1}$ is completely continuous, and this is why a locally convex space $\mathcal{P}$ is the space of type $\left(L N^{*}\right)$; in particular, it is complete, separated, metrizable, reflexive, Montel space (cf. [1]). Moreover, in this space, the multiplication by an independent variable $z$ is continuous, i.e., $\mathcal{P}$ is a topological module over the ring of polynomials $\mathbb{C}[z]$.

In the present paper we study some special properties of closed submodules of module $\mathcal{P}$. For the sake of brevity we shall make use of the notion "submodule" meaning a closed submodule. The study of submodules in $\mathcal{P}$ is an interesting issue because they are dual to closed subspaces of space $C^{\infty}(a ; b)$ invariant w.r.t. the differentiation.

For a function $\varphi \in \mathcal{P}$ and each $\lambda \in \mathbb{C}$ we define its divisor

$$
n_{\varphi}(\lambda)=\left\{\begin{array}{l}
0, \quad \text { if } \varphi(\lambda) \neq 0, \\
m, \quad \text { if } \lambda \text { is a zero of } \varphi \text { of multiplicity } m .
\end{array}\right.
$$

A divisor of submodule $\mathcal{J} \subset \mathcal{P}$ is called the function $n_{\mathcal{J}}(\lambda)=\min _{\varphi \in \mathcal{J}} n_{\varphi}(\lambda)$. We denote by $\Lambda_{\varphi}=\left\{\left(\lambda_{k}, m_{k}\right): m_{k}=n_{\varphi}\left(\lambda_{k}\right)>0, k=1,2, \ldots\right\}$ the zero set of function $\varphi \in \mathcal{P}$ being not

N.F. Abuzyarova, Closed submodules in the module of Entire Functions of Exponential type AND POLYNOMIAL GROWTH ON THE REAL AXIS.

(c) Abuzyarova N.F. 2014.

The work is supported by grant no. 01201456408 of Ministry of Education and Science of Russian Federation.

Submitted May 16, 2014. 
identically zero; $\Lambda_{\mathcal{J}}=\left\{\left(\lambda_{k}, m_{k}\right): m_{k}=n_{\mathcal{J}}\left(\lambda_{k}\right)>0, k=1,2, \ldots\right\}$ is the zero set of submodule $\mathcal{J} \neq\{0\}$.

It is known (see, for instance, [2]) that each element of space $\mathcal{P}$ is a function of completely regular growth of order 1 whose indicator diagram is the segment of imaginary axis $\left[\mathrm{i} c_{\varphi} ; \mathrm{i} d_{\varphi}\right] \subset$ $(\mathrm{i} a ; \mathrm{i} b)$. For a submodule $\mathcal{J}$ we let $c_{\mathcal{J}}=\inf _{\varphi \in \mathcal{J}} c_{\varphi}, d_{\mathcal{J}}=\sup _{\varphi \in \mathcal{J}} d_{\varphi}$. We call the set $\left[c_{\mathcal{J}} ; d_{\mathcal{J}}\right]$ indicator segment of submodule $\mathcal{J}$.

Since $\mathcal{P}$ is a space of type $\left(L N^{*}\right)$, a set $B \subset P$ is bounded if and only if it is contained and bounded in one of Banach spaces $P_{k}$ (see [1, Thm. 2]). Employing this fact and the definition of topology in $\mathcal{P}$, it is easy to make sure that space $\mathcal{P}$ is bornological and $b$-stable. We recall that a locally convex space of entire functions is called $b$-stable if for each bounded set $B \subset \mathcal{P}$ the set of all all entire functions $\psi$ reading as $\psi(z)=\varphi(z) /(z-\lambda), \lambda \in \mathbb{C}, \varphi \in B$, is contained and bounded in $\mathcal{P}$ (see [3, Sect. 1]).

By the said above, to study the submodules in module $\mathcal{P}$ one can apply abstract methods developed by I.F. Krasičkov-Ternovskiǔ in [3], 4].

A submodule $\mathcal{J}$ is weakly localizable if it contains all the functions $\varphi \in \mathcal{P}$ satisfying the conditions 1) $\left.n_{\varphi}(z) \geqslant n_{\mathcal{J}}(z), z \in \mathbb{C} ; 2\right)$ the indicator diagram of function $\varphi$ is contained in the set $\mathrm{i}\left[c_{\mathcal{J}} ; d_{\mathcal{J}}\right]$. If $c_{\mathcal{J}}=a$ and $d_{\mathcal{J}}=b$, the weak localizability of $\mathcal{J}$ means that this submodule is local or admits local description (shortly, it is localizable).

A submodule $\mathcal{J}$ is called stable at a point $\lambda \in \mathbb{C}$ if the conditions $\varphi \in \mathcal{J}$ and $n_{\varphi}(\lambda)>n_{\mathcal{J}}(\lambda)$ imply $\varphi /(z-\lambda) \in \mathcal{J}$. A submodule $\mathcal{J}$ is stable if it is stable at each point $\lambda \in \mathbb{C}$.

The notions "stable (at a point) submodule", "local module" were introduced in [3], [5].

It is clear that stability of submodule $\mathcal{J}$ is a necessary condition of its weak localizability.

We denote by $\mathcal{J}_{\varphi_{1}, \ldots, \varphi_{m}}$ the closed submodule generated by functions $\varphi_{1}, \ldots, \varphi_{m} \in \mathcal{P}$ :

$$
\mathcal{J}=\overline{\left\{p_{1} \varphi_{1}+\cdots+p_{m} \varphi_{m}, \quad p_{1}, \ldots, p_{m} \in \mathbb{C}[z]\right\}}
$$

Functions $\varphi_{1}, \ldots, \varphi_{m}$ are called generators of submodule $\mathcal{J}_{\varphi_{1}, \ldots, \varphi_{m}}$.

It follows from the results of work [4, Sect. 4] that the main (generated by one function) submodule in $\mathcal{P}$ is always stable. One can check it also straightforwardly taking into consideration that module $\mathcal{P}$ is pointwise stable (pointwise stability of $\mathcal{P}$ is discussed in details in the proof of Theorem 1). In contrast to main submodules, those generated by $m$ functions, $m>1$, are not always stable. For instance, the submodule generated in $\mathcal{P}$ by the functions $e^{-\mathrm{i} c z}, e^{-\mathrm{i} d z}$, where $a<c<d<b$, is unstable by Proposition 1 and 2 of the present work and Example 2 in work [12, § 2].

In what follows, in Section 3, we consider the issue on conditions ensuring the stability of submodule generated in $\mathcal{P}$ by two functions $\varphi, \psi$ in terms of mutual location of zeroes (and thus, in terms of "close" growth) of these functions (Theorem 1). This study is based on the stability criterion for submodule with finite amount of generators obtained by I.F. Krasičkov-Ternovskiı ([4, Prop. 4.9]).

The sufficient condition for stability of submodule with two generators provided in Theorem 11 seem to be far from necessary as in all statements of this kind we know (cf. [6]-[11]). On the other hand, in contrast to the general criterion of I.F. Krasičkov-Ternovskil [4, Prop. 4.9], the stability condition formulated in terms of mutual location of zero sets of two functions (or submodules) are observable and checkable. In particular, it allows one to obtain statements on 2-generated or representation by a sum of two special local submodules (ideals) for local submodules (cf. [6]-[11]), or sometimes, like in our case, only for stable submodules (Theorem 2).

The example of unstable submodule provided above shows that 2-generated submodule in $\mathcal{P}$ is not necessarily main. Theorem 2 implies even more: not each stable submodule with two generators in $\mathcal{P}$ is main. 
We note that for a wide class of weight modules of functions holomorphic in a domain $\Omega \subset \mathbb{C}$, in work [9] there were studied the conditions of locality (stability) of a submodule generated by two local (stable) submodules in terms of "closeness" of zero sequences for generating submodules. However, the gaps between the weights determining the topology of modules considered in [9] grows faster than the logarithmic function and this is why here the results of this work are not applicable since in the module the gaps between the weights are always logarithmic.

\section{Duality issues}

2.1. Duality principle. Let $\mathcal{E}=C^{\infty}(a ; b)$ be the Schwarz space equipped with a standard topology of projective limit of Banach spaces $C^{k}\left[a_{k} ; b_{k}\right]$. It is known that $\mathcal{E}$ is a complete metrizable reflexive locally convex space in which each bounded space is relatively compact. Let $D=\frac{\mathrm{d}}{\mathrm{d} t}$ be the operator of differentiation, $W \subset \mathcal{E}$ be a closed and invariant w.r.t $D$ (shortly, $D$-invariant) subspace: $D W \subset W$. If else is not said, we assume that $W \neq \mathcal{E}$. We denote by $\operatorname{Exp} W$ the set of all root elements of operator $D$ (the exponential monomials $t^{j} e^{-\mathrm{i} \lambda t}$ ) contained in $W$.

In work [12] the following problem on spectral synthesis was solved: the spectrum $\sigma(W)$ of restriction of differentiation operator on $D$-invariant subspace $W$ (called also spectrum of $D$-invariant subspace $W)$ was either discrete or it coincided with the complex plane $([12$, Thm. 2.1]). In the former case $\sigma(W)$ is a sequence of multiple points $\Lambda=\left\{\left(-\mathrm{i} \lambda_{j}, m_{j}\right),\right\} m_{j} \in \mathbb{N}$, $j=1,2, \ldots$, at that, $\operatorname{Exp} W=\left\{t^{k} e^{-\mathrm{i} \lambda_{j} t}, k=0,1, \ldots, m_{j}-1, j=1,2, \ldots\right\}$.

Let $I \subset(a ; b)$ be a relatively closed in $(a ; b)$ non-empty interval. We let

$$
W_{I}=\left\{f \in \mathcal{E}: \quad f^{(k)}(t)=0, t \in I, k=0,1,2, \ldots\right\} .
$$

It was observed in work [12, Sect. 2, Ex. 1] that in the case $I=\{c\}, c \in(a ; b)$ the corresponding $D$-invariant subspace $W_{c}$ is a set of all functions $f \in \mathcal{E}$ satisfying the condition $f^{(k)}(c)=0$, $k=0,1, \ldots$, and it has the empty spectrum.

It was also proven in [12] that for each $D$-invariant subspace $W \neq \mathcal{E}$ there exists a minimal relatively closed in $(a ; b)$ interval $I \neq \emptyset$ obeying the inclusion $W_{I} \subset W$ (Theorem 4.1).

We denote this interval by $I_{W}$ and we let $c_{W}=\inf \left\{t \in I_{W}\right\}, d_{W}=\sup \left\{t \in I_{W}\right\}$.

In accordance with Paley-Wiener-Schwarz theorem [13, Ch. 7], the Fourier-Laplace transform $\mathcal{F}$ makes a linear topological isomorphism between a strongly dual to $\mathcal{E}$ space $\mathcal{E}^{\prime}$ and space $\mathcal{P}$ :

$$
S \in \mathcal{E}^{\prime} \leftrightarrow \varphi \in \mathcal{P} \Longleftrightarrow \varphi=\mathcal{F}(S)=\left(S, e^{-\mathrm{i} t z}\right) .
$$

By the symbol ch $\operatorname{supp} S$ we indicate the convex hull of the support of a functional $S \in \mathcal{E}^{\prime}$. Since all the elements in space $\mathcal{E}^{\prime}$ have compact supports, ch $\operatorname{supp} S$ is an interval in $(a ; b)$.

Proposition 1. (Duality principle.) Between D-invariant subspaces $W \subset \mathcal{E}$ and closed submodules $\mathcal{J} \subset \mathcal{P}$ there is the one-to-one correspondence described by the rule: $W \leftrightarrow \mathcal{J} \Longleftrightarrow$ $\mathcal{J}=\mathcal{F}\left(W^{0}\right)$, where $W^{0}=\left\{S \in \mathcal{E}^{\prime}: \quad(S, f)=0, \quad f \in W\right\}$ is the annihilating subspace for $W$. At that,

$$
\operatorname{Exp} W=\left\{t^{j} e^{-i \lambda_{k} t}, j=0, \ldots m_{k}-1, \quad\left(\lambda_{k}, m_{k}\right) \in \Lambda_{\mathcal{J}}\right\}
$$

and the boundary of interval $I_{W}$ are points $c_{\mathcal{J}}$ and $d_{\mathcal{J}}$.

Proof. The first part of the duality principle is proven by the same arguments as in [5, Sect. 2] and it implies (2.2).

Let us prove the statement on the boundary of interval $I_{W}$. Let $\mathcal{J}=\mathcal{F}\left(W^{0}\right)$ be the annihilating submodule of $D$-invariant subspace $W$. Letting $I^{\prime}=(a ; b) \bigcap\left[c_{\mathcal{J}} ; d_{\mathcal{J}}\right]$, we see that $D$-invariant subspace $W_{I^{\prime}}$ is annihilated by all the functionals in subspace $W^{0}$. By the first part of the duality principle we conclude that $W_{I^{\prime}}$ is contained in $W$ and thus $W_{I^{\prime}} \subset W_{I_{W}}$. The latter is equivalent to $I^{\prime} \supset I_{W}$. Suppose that it is proper, for instance, $c_{\mathcal{J}}<c_{W}$. Then by Paley-Wiener-Schwarz theorem, in annihilating subspace $W^{0}$ there exists a distribution $S$ 
possessing the following property: the intersection of the support of $S$ and the open interval $\left(c_{\mathcal{J}} ; c_{W}\right)$ is non-empty. By the definition of the support of distribution, there exists an infinitely differentiable function $\varphi_{0}$ such that $\operatorname{ch} \operatorname{supp} \varphi_{0} \Subset\left(c_{\mathcal{J}} ; c_{W}\right)$ and $\left(S, \varphi_{0}\right) \neq 0$. Therefore, $\varphi_{0} \notin W$. On the other hand, we see that $\varphi_{0} \in W_{I_{W}} \subset W$. Hence, the relation $c_{\mathcal{J}}<c_{W}$ is impossible.

In the same way one can prove that the strict inequality $d_{\mathcal{J}}>d_{W}$ is impossible.

D-invariant subspace $W$ happens to have discrete spectrum if and only if its annihilating submodule $\mathcal{J}$ is stable.

The necessary part of this statement is contained in Item ii) of Proposition 3.1 [12], while the sufficient part is provided by the following proposition.

Proposition 2. If annihilating submodule $\mathcal{J} \neq\{0\}$ of $D$-invariant subspace $W$ is stable, then $W$ has a discrete spectrum $\sigma_{W}=-i \Lambda_{\mathcal{J}}$.

Proof. Thanks to 2.2 we need to prove only $\sigma_{W} \subset-\mathrm{i} \Lambda_{\mathcal{J}}$.

By proposition 2.2 in work [12], point $\lambda$ lies in a set $\mathbb{C} \backslash \sigma_{W}$ if and only if the identity $(D-\lambda) W=W$ holds true. This identity means that the mapping

$$
(D-\lambda): W \rightarrow W
$$

is surjective.

First we consider the case when $\mathcal{J}=\mathcal{J}_{\varphi}$ is the main submodule generated by the function $\varphi=\mathcal{F}(S), S \in \mathcal{E}^{\prime}$; in this case $\Lambda_{\mathcal{J}}=\Lambda_{\varphi}$. We denote by $W_{\varphi}$ the corresponding $D$-invariant subspace (for which $\mathcal{F}\left(W_{\varphi}^{0}\right)=\mathcal{J}_{\varphi}$ ). Subspace $W_{\varphi}$ consists of all the functions $f \in \mathcal{E}$, satisfying the identities

$$
\left(S, D^{k} f\right)=0, \quad k=0,1,2, \ldots
$$

Let us prove that for each point $\lambda_{0} \in \mathbb{C} \backslash \Lambda_{\varphi}$ the identity

$$
\left(D+\mathrm{i} \lambda_{0}\right) W_{\varphi}=W_{\varphi}
$$

holds true.

We suppose that $\varphi\left(\lambda_{0}\right)=1$.

Let ch $\operatorname{supp} S=[c ; d] \subset(a ; b)$. For $f \in W_{\varphi}$ we define

$$
\tilde{f}(t)=-\left(S, \int_{c}^{t} f(\tau) e^{-\mathrm{i}(t-\tau) \lambda_{0}} \mathrm{~d} \tau\right) e^{-\mathrm{i} t \lambda_{0}}+\int_{c}^{t} f(\tau) e^{-\mathrm{i}(t-\tau) \lambda_{0}} \mathrm{~d} \tau .
$$

It is easy to check that $\left(D+\mathrm{i} \lambda_{0}\right) \tilde{f}=f$ and $\left(S, D^{k} \tilde{f}\right)=0, k=0,1,2, \ldots$ Thus, $\tilde{f}$ is a solution to equation $\left(D+\mathrm{i} \lambda_{0}\right) g=f, f \in W_{\varphi}$, belonging to space $W_{\varphi}$.

Relation (2.3) and, therefore, the inclusion $\sigma_{W_{\varphi}} \subset-\mathrm{i} \Lambda_{\mathcal{J}_{\varphi}}$ for the main submodule $\mathcal{J}_{\varphi}$ are proven.

Now we consider the case when $\mathcal{J}=\mathcal{F}\left(W^{0}\right)$ is an arbitrary stable submodule $\mathcal{P}, \lambda_{0} \notin \Lambda_{\mathcal{J}}$. Let $\varphi_{0} \in \mathcal{J}, \varphi_{0}\left(\lambda_{0}\right)=1$.

If $\psi \in \mathcal{J}$, then the function

$$
\Psi=\left\{\begin{array}{l}
\psi-\frac{\psi\left(\lambda_{0}\right)}{\lambda_{0}} z \varphi_{0}, \quad \lambda_{0} \neq 0 \\
\psi-\psi(0) \varphi_{0}, \quad \lambda_{0}=0
\end{array}\right.
$$

belongs to $\mathcal{J}$ and vanishes at point $\lambda_{0}$. Thus, $\tilde{\psi}=\Psi /\left(z-\lambda_{0}\right) \in \mathcal{J}$. Hence, the representation

$$
\psi=\left\{\begin{array}{l}
\left(z-\lambda_{0}\right) \tilde{\psi}+\frac{\psi\left(\lambda_{0}\right)}{\lambda_{0}} z \varphi_{0}, \quad \lambda_{0} \neq 0 \\
z \tilde{\psi}+\psi(0) \varphi_{0}, \quad \lambda_{0}=0
\end{array}\right.
$$

holds true for an arbitrary function $\psi \in \mathcal{J}$. For submodule $\mathcal{J}$ we can write

$$
\mathcal{J}=\left(z-\lambda_{0}\right) \mathcal{J}+\mathcal{J}_{\varphi_{0}} .
$$


Employing duality principle (Proposition 1) and the reflexivity of space $\mathcal{E}$, by (2.5) it easy to obtain that the original $D$-invariant subspace $W$ is the intersection of $D$-invariant subspaces $W_{1}$ and $W_{2}$ having annihilating submodules $\left(z-\lambda_{0}\right) \mathcal{J}$ and $\mathcal{J}_{\varphi_{0}}$, respectively.

A solution in $W$ to equation $\left(D+\mathrm{i} \lambda_{0}\right) g=f, f \in W$, is function $\tilde{f}$ determined by formula (2.4). Indeed, as it has been observed above, $\tilde{f} \in W_{2}$, and identity $\left(D+\mathrm{i} \lambda_{0}\right) \tilde{f}=f \in W$ is equivalent to $\tilde{f} \in W_{1}$. This is why $\tilde{f} \in W_{1} \cap W_{2}=W$. Hence, $\left(D+\mathrm{i} \lambda_{0}\right): W \rightarrow W$ is a surjective operator. In accordance with the proposition in work [12] cited in the beginning of the proof, the point $\left(-\mathrm{i} \lambda_{0}\right)$ does not belong to spectrum $\sigma_{W}$. Thus, we have the inclusion $\mathbb{C} \backslash\left(-\mathrm{i} \Lambda_{\mathcal{J}}\right) \subset \mathbb{C} \backslash \sigma_{W}$ which is equivalent to the desired one.

2.2. Preserving of class $\mathcal{F}\left(C_{0}^{\infty}(a ; b)\right)$ under perturbation of zeroes. We consider the function

$$
\varphi(z)=\int_{a}^{b} s(t) e^{-\mathrm{i} t z} \mathrm{~d} t, \quad s \in C_{0}^{\infty}(a ; b)
$$

and by $\Lambda=\left\{\lambda_{k}\right\}, k=1,2, \ldots$ we denote the sequence of its zeroes taken in the ascending order of absolute values: $\left|\lambda_{1}\right| \leqslant\left|\lambda_{2}\right| \leqslant \ldots$

We are interesting in the conditions of closeness of another sequence $\Gamma=\left\{\gamma_{k}\right\}$ to sequence $\Lambda$, under which $\Gamma$ is also the set of zeroes of a function $\psi$ in $\mathcal{F}\left(C_{0}^{\infty}(a ; b)\right)$.

The stability for various classes of functions of compactly supported Fourier transforms w.r.t. the shifts of zeroes was studied by A.M. Sedletskii [14].

Let $\left(a^{\prime} ; b^{\prime}\right) \Subset \mathbb{R}$. Theorem 5.1.2 in work [14] contains, in particular, the following statement: The condition

$$
\sum_{j} \frac{\left|\lambda_{j}-\gamma_{j}\right|}{1+\left|\operatorname{Im} \lambda_{j}\right|+\left|\operatorname{Im} \gamma_{j}\right|} \leqslant+\infty
$$

preserves the class $\mathcal{F}\left(L^{p}\left(a^{\prime} ; b^{\prime}\right)\right), 1 \leqslant p \leqslant \infty$.

Proposition 3. Let $\varphi$ be a function determined by (2.6) with a sequence of zeroes $\Lambda=\left\{\lambda_{k}\right\}$, $0<\left|\lambda_{1}\right| \leqslant\left|\lambda_{2}\right| \leqslant \ldots$, and let $\Gamma=\left\{\gamma_{k}\right\}$ be another sequence so close to $\Lambda$ that condition (2.7) is satisfied. Then for each $a^{\prime}, b^{\prime} \in \mathbb{R}$ such that ch supp $s \Subset\left(a^{\prime} ; b^{\prime}\right) \subset(a ; b)$, function $\psi$ defined by the formula

$$
\psi(z)=e^{-i c z} \lim _{R \rightarrow \infty} \prod_{\left|\gamma_{k}\right|<R}\left(1-z / \gamma_{k}\right), \quad \text { where } \quad c=\left(h_{\varphi}(\pi / 2)+h_{\varphi}(-\pi / 2)\right) / 2,
$$

belongs to the class $\mathcal{F}\left(C_{0}^{\infty}\left(a^{\prime} ; b^{\prime}\right)\right)$ and its indicator $h_{\psi}$ coincides with the indicator $h_{\varphi}$ of function $\varphi$.

Proof. Let $a^{\prime}, b^{\prime} \in \mathbb{R}$ be as in the formulation of the proposition. By the result of A.M. Sedletskii cited above, function $\psi$ is the image of the Fourier-Laplace transform of some function $\tilde{s} \in$ $L^{q}\left(a^{\prime} ; b^{\prime}\right)$ for each $1 \leqslant q \leqslant \infty$. At that, as one can see in the proofs of Theorems 5.1.1, 5.1.2 in work [14], $\operatorname{ch} \operatorname{supp} \tilde{s}=\operatorname{ch} \operatorname{supp} s$, and thus indicators of entire functions $\varphi$ and $\psi$ coincide.

Applying similar arguments to functions $z^{m} \varphi$ and $z^{m} \psi, m=1,2, \ldots$, and bearing in mind the identities

$$
z^{m} \varphi=\mathcal{F}\left(s^{(m)}\right), \quad z^{m} \psi=\mathcal{F}\left(\tilde{s}^{(m)}\right) \quad\left(\tilde{s}^{(m)} \text { is the generalized derivative of a distribution } \tilde{s}\right),
$$

we obtain

$$
\tilde{s}^{(m)} \in L^{q}\left(a^{\prime} ; b^{\prime}\right), \quad 1 \leqslant q \leqslant \infty, \quad \text { and } \quad \operatorname{chsupp} \tilde{s}^{(m)} \subset \operatorname{chsupp} s, m=0,1, \ldots
$$

Thus, $\tilde{s} \in C_{0}^{\infty}\left(a^{\prime} ; b^{\prime}\right), \psi=\mathcal{F}(\tilde{s}) \in \mathcal{F}\left(C_{0}^{\infty}\left(a^{\prime} ; b^{\prime}\right)\right)$. 
It was shown in work [14] (proof of Theorem 5.1.2) that condition (2.7) the convergence of the series $\sum_{j=1}^{\infty} \frac{\left|\lambda_{j}-\gamma_{j}\right|}{1+\left|\operatorname{Im} \lambda_{j}\right|}$. We denote its sum by $C$.

The following statement complementing Proposition 3 will be employed in the proof of Theorem 2 .

Lemma 1. Under the hypothesis of Proposition 3 the inequalities

$$
\max _{a^{\prime} \leqslant t \leqslant b^{\prime}}\left|\tilde{s}^{(m)}(t)\right| \leqslant A_{m}, m=0,1, \ldots, \quad \text { where } \quad A_{m}=e^{2 C}\left\|s^{(m+1)}\right\|_{L^{1}\left(a^{\prime} ; b^{\prime}\right)}
$$

hold true.

Proof. We let

$$
s_{m, 0}(t)=s^{(m)}(t), \quad s_{m, n}(t)=s_{m, n-1}(t)-\mathrm{i}\left(\gamma_{n}-\lambda_{n}\right) \int_{a^{\prime}}^{t} e^{\mathrm{i} \lambda_{n}(t-\tau)} s_{m, n-1}(\tau) \mathrm{d} \tau, \quad t \in\left(a^{\prime} ; b^{\prime}\right) .
$$

Estimate (5.1.14) in [14], the definitions of functions $s_{m, n}$ and quantity $C$ yield that

$$
\left\|s_{m, n}\right\|_{L^{1}\left(a^{\prime} ; b^{\prime}\right)} \leqslant\left(1+\frac{2\left|\lambda_{j}-\gamma_{j}\right|}{1+\left|\operatorname{Im} \lambda_{j}\right|}\right)\left\|s_{m, n-1}\right\|_{L^{1}\left(a^{\prime} ; b^{\prime}\right)} \leqslant \cdots \leqslant e^{2 C}\left\|s^{(m+1)}\right\|_{L^{1}\left(a^{\prime} ; b^{\prime}\right)}
$$

Since sequence $s_{m, n}$ converges to $\tilde{s}^{(m)}$ in $L^{1}\left(a^{\prime} ; b^{\prime}\right)$ (that was proven in Theorem 5.1.2 in work [14]), by the latter inequality we conclude that

$$
\left\|\tilde{s}^{(m)}\right\|_{L^{1}\left(a^{\prime} ; b^{\prime}\right)} \leqslant e^{2 C}\left\|s^{(m)}\right\|_{L^{1}\left(a^{\prime} ; b^{\prime}\right)} .
$$

By this estimate we obtain desired inequalities $(2.9)$ :

$$
\max _{a^{\prime} \leqslant t \leqslant b^{\prime}}\left|\tilde{s}^{(m)}(t)\right|=\max _{a^{\prime} \leqslant t \leqslant b^{\prime}}\left|\int_{a}^{t} \tilde{s}^{(m+1)}(\tau) \mathrm{d} \tau\right| \leqslant\left\|\tilde{s}^{(m+1)}\right\|_{L^{1}\left(a^{\prime} ; b^{\prime}\right)} \leqslant e^{2 C}\left\|s^{(m+1)}\right\|_{L^{1}\left(a^{\prime} ; b^{\prime}\right)} .
$$

\section{Sufficient CONDitions FOR STABILITy OF 2-GENERATED SUBMOdUle IN $\mathcal{P}$}

3.1. Auxiliary estimates. Let $\varphi \in \mathcal{P}, \varphi(0)=1, \Lambda=\left\{\lambda_{j}\right\},\left|\lambda_{1}\right| \leqslant\left|\lambda_{2}\right| \leqslant \ldots$ be the set of zeroes for function $\varphi$. It is known [2, Ch. II] that $\varphi$ satisfies the representation

$$
\varphi(z)=e^{-\mathrm{i}\left(c_{\varphi}+d_{\varphi}\right) z / 2} \prod_{j=1}^{\infty}\left(1-\frac{z}{\lambda_{j}}\right), \quad \text { where } c_{\varphi}=h(-\pi / 2), \quad d_{\varphi}=h(\pi / 2),
$$

at that, the infinite product converges conditionally and uniformly on compact sets in $\mathbb{C}$, and sequence $\Lambda$ has the density $\Delta_{0}=\left(d_{\varphi}-c_{\varphi}\right) / 2 \pi$.

We consider one more function $\psi \in \mathcal{P}, \psi(0)=1$, with zero set $\Gamma=\left\{\gamma_{j}\right\}$ taken in the order of ascending absolute values $\left|\gamma_{j}\right|$ and having the density $\Delta_{0}$. Function $\psi$ also satisfies the representation (3.1) with $\gamma_{j}$ instead of $\lambda_{j}$.

We introduce needed notations.

$$
\begin{array}{ll}
\varphi_{k}(z)=\prod_{j=1}^{k}\left(1-\frac{z}{\lambda_{j}}\right), & \psi_{k}(z)=\prod_{j=1}^{k}\left(1-\frac{z}{\gamma_{j}}\right), \\
\Phi_{M}(z)=\prod_{j \in M}\left(1-\frac{z}{\lambda_{j}}\right), & \Psi_{M}(z)=\prod_{j \in M}\left(1-\frac{z}{\gamma_{j}}\right),
\end{array}
$$

where $M \subset \mathbb{N}$ is a non-empty set for which both the products converge (conditionally and uniformly on compact sets in $\mathbb{C}$ ); if $M=\emptyset$, we let $\Phi_{M}(z)$ and $\Psi_{M}(z)$ to be identically one.

For numbers $\sigma \in(0 ; 1 / 2)$ and $\lambda \in \mathbb{C}$ we denote by $e_{\sigma}(\lambda)$ the closed circle of radius $\sigma|\lambda|$ centered at $\lambda$, and for a non-empty set $M \subset \mathbb{N}$ we let $E_{M, \sigma}=\bigcup_{j \in M}\left(e_{\sigma}\left(\lambda_{j}\right) \bigcup e_{\sigma}\left(\gamma_{j}\right)\right)$. 
Let

$$
\chi(\mu)=\frac{1}{\mu} \ln (1+\mu)+\ln \left(1+\frac{1}{\mu}\right) .
$$

This function strictly monotonously decreases on the positive semi-axis and takes positive values. Thus, there exists the inverse function $\mu(\chi)>0$ also strictly monotonously decreasing for positive $\chi$.

The following quantities characterize the closeness of sequences $\Lambda$ and $\Gamma$.

$$
S_{n}=\sum_{j \geqslant n}\left|\frac{1}{\lambda_{j}}-\frac{1}{\gamma_{j}}\right|, \quad K_{M}=\max _{j \in M}\left\{\left|\frac{\lambda_{j}}{\gamma_{j}}\right|,\left|\frac{\gamma_{j}}{\lambda_{j}}\right|\right\}, \quad M \subset \mathbb{N}, \quad M \neq \emptyset,
$$

we let $K_{M}=1$ if $M=\emptyset$.

In what follows in this subsection we assume that

$$
\left|\lambda_{j}\right| \geqslant 2, \quad,\left|\gamma_{j}\right| \geqslant 2, \quad j=1,2, \ldots,
$$

for some number $\Delta>\Delta_{0}$ the inequalities

$$
n_{\Lambda}(r)<\Delta r, \quad n_{\Gamma}(r)<\Delta r
$$

hold true for each $r>0$. Here $n_{\Lambda}(r)=\sum_{j:\left|\lambda_{j}\right| \leqslant r} 1$ and $n_{\Gamma}(r)=\sum_{j:\left|\gamma_{j}\right| \leqslant r} 1$ are counting function for sequences $\Lambda$ and $\Gamma$.

Lemma 2. 1. Let $\delta>0$ and $\Delta>\Delta_{0}$ is a number for which the inequalities (3.4) hold true. For each $k$ as $|z| \geqslant \max \left\{2, \mu(\delta / \Delta) \max \left\{\left|\lambda_{k}\right|,\left|\gamma_{k}\right|\right\}\right\}$ the estimates

$$
\ln \left|\varphi_{k}(z)\right| \leqslant \min \{\delta|z|, k \ln |z|\}, \quad \ln \left|\psi_{k}(z)\right| \leqslant \min \{\delta|z|, k \ln |z|\}
$$

are valid.

2. For $\sigma \in(0 ; 1 / 2), M \subset \mathbb{N}$ the inequalities

$$
\begin{aligned}
& |\ln | \frac{\Phi_{M}(z)}{\Psi_{M}(z)}|| \leqslant \frac{S_{p}}{\sigma}|z|, \\
& \left|1-\frac{\Phi_{M}(z)}{\Psi_{M}(z)}\right| \leqslant \frac{\sqrt{\pi^{2}+1}}{\sigma} S_{p}|z| \exp \left(\frac{\sqrt{\pi^{2}+1}}{\sigma} S_{p}|z|\right)
\end{aligned}
$$

hold true outside set $E_{M, \sigma}$, where $p=\min \{j: j \in M\}$, and set $M \subset \mathbb{N}$ is so that the products determining functions $\Phi_{M}, \Psi_{M}$ converge.

3. Let function $\varphi$ satisfies inequality

$$
\ln |\varphi(z)| \leqslant \frac{d_{\varphi}-c_{\varphi}}{2}|\operatorname{Im} z| .
$$

for each $z \in \mathbb{C}$. For arbitrary $\sigma \in(0 ; 1 / 2), R>0, k \in \mathbb{N}$ we let

$$
M=M(k, R, \sigma)=\left\{j \in \mathbb{N}: j>k,\left(e_{\sigma}\left(\lambda_{j}\right) \bigcup e_{\sigma}\left(\gamma_{j}\right)\right) \bigcap[-R, R] \neq \emptyset\right\} .
$$

Then inequality

$$
\begin{aligned}
&\left|\psi_{k}(x) \varphi(x)-\varphi_{k}(x) \psi(x)\right| \leqslant \frac{\sqrt{\pi^{2}+1}(1+\sigma)}{\sigma(1-\sigma)} K_{M} S_{k+1} R \\
& \cdot \exp \left[\left(\frac{\sqrt{\pi^{2}+1}(1+\sigma)}{\sigma(1-\sigma)} K_{M} S_{k+1}+\frac{\sigma\left(d_{\varphi}-c_{\varphi}\right)}{1-\sigma}\right) R+\min \{\delta R, k \ln R\}\right] \\
& \cdot\left(1+\exp \left(\frac{\sqrt{\pi^{2}+1}(1+\sigma)}{\sigma(1-\sigma)} K_{M} S_{k+1} R\right)\right)
\end{aligned}
$$

holds true for each $x \in[-R ; R], R \geqslant \max \left\{2, \mu(\delta / \Delta)\left|\lambda_{k}\right|\right\}, k \in \mathbb{N}$. 
We observe that quantities $S_{p}$ in the right hands of inequalities (3.6), (3.7) and $S_{k+1}$ in the right hand side of inequality 3.10 can take value $+\infty$; in this case these inequalities become trivial. In what follows, while using these inequalities, on sequences $\Lambda$ and $\Gamma$ we impose conditions ensuring the finiteness of $S_{p}$.

Proof. 1. Inequalities $\ln \left|\varphi_{k}(z)\right| \leqslant \delta|z|, \ln \left|\psi_{k}(z)\right| \leqslant \delta|z|$ for $|z| \geqslant \mu(\delta / \Delta) \max \left\{\left|\lambda_{k}\right|,\left|\gamma_{k}\right|\right\}$ can be proven in the same way as Item 1 of Lemma 1 in [6] taking into consideration conditions (3.4) for number $\Delta$ and ordering of sequences $\Lambda$ and $\Gamma$ by ascending $\left|\lambda_{j}\right|,\left|\gamma_{j}\right|$.

By conditions (3.3) we obtain immediately that $\ln \left|\varphi_{k}(z)\right| \leqslant k \ln |z|, \ln \left|\psi_{k}(z)\right| \leqslant k \ln |z|$ as $|z| \geqslant 2$.

2. To prove estimates (3.6) and (3.7) we make use the scheme employed in the proof of Items 2 and 3 of Lemma 1 in [6]. We fix arbitrary $\sigma \in(0 ; 1 / 2)$. For $z \notin e_{\sigma}\left(\gamma_{j}\right)$ we have

$$
\ln \left|\frac{1-z / \lambda_{j}}{1-z / \gamma_{j}}\right|=\ln \left|1+\frac{\left(1 / \gamma_{j}-1 / \lambda_{j}\right) z}{1-z / \gamma_{j}}\right| \leqslant\left|\frac{1}{\gamma_{j}}-\frac{1}{\lambda_{j}}\right| \frac{|z|}{\sigma} .
$$

In the same way, for $z \notin e_{\sigma}\left(\lambda_{j}\right)$ we get

$$
\ln \left|\frac{1-z / \gamma_{j}}{1-z / \lambda_{j}}\right| \leqslant\left|\frac{1}{\gamma_{j}}-\frac{1}{\lambda_{j}}\right| \frac{|z|}{\sigma} .
$$

These inequalities imply estimate 3.6 for each $z$ outside set $E_{M, \sigma}$ :

$$
|\ln | \frac{\Phi_{M}(z)}{\Psi_{M}(z)}|| \leqslant \sum_{j \in M}|\ln | \frac{1-z / \lambda_{j}}{1-z / \gamma_{j}}|| \leqslant\left(\sum_{j \in M}\left|\frac{1}{\gamma_{j}}-\frac{1}{\lambda_{j}}\right|\right) \frac{|z|}{\sigma} \leqslant \frac{S_{p}}{\sigma}|z| .
$$

In order to obtain inequality 3.7 , first we estimate the expression $\left|\ln \frac{\Phi_{M}(z)}{\Psi_{M}(z)}\right|$ outside set $E_{M, \sigma}$.

We observe that

$$
\left|\operatorname{Re} \ln \frac{\Phi_{M}(z)}{\Psi_{M}(z)}\right|=|\ln | \frac{\Phi_{M}(z)}{\Psi_{M}(z)}||,\left|\operatorname{Im} \ln \frac{\Phi_{M}(z)}{\Psi_{M}(z)}\right|=\left|\arg \frac{\Phi_{M}(z)}{\Psi_{M}(z)}\right| .
$$

For $\left|\operatorname{Re} \ln \frac{\Phi_{M}(z)}{\Psi_{M}(z)}\right|$ inequality 3.6 holds true.

Let us estimate $\left|\operatorname{Im} \ln \frac{\Phi_{M}(z)}{\Psi_{M}(z)}\right|$. In order to do it, we employ the easily checked inequality

$$
\arg (1+w) \leqslant \pi|w|, \quad w \in \mathbb{C}
$$

where the branch of the function arg takes the values in the interval $(-\pi ; \pi]$.

For $z \notin e_{\sigma}\left(\gamma_{j}\right)$ we have

$$
\arg \left|\frac{1-z / \lambda_{j}}{1-z / \gamma_{j}}\right|=\arg \left|1+\frac{\left(1 / \gamma_{j}-1 / \lambda_{j}\right) z}{1-z / \gamma_{j}}\right| \leqslant \frac{\pi}{\sigma}\left|\frac{1}{\gamma_{j}}-\frac{1}{\lambda_{j}}\right||z| .
$$

In the same way, for $z \notin e_{\sigma}\left(\lambda_{j}\right)$ we obtain

$$
\arg \left|\frac{1-z / \gamma_{j}}{1-z / \lambda_{j}}\right| \leqslant \frac{\pi}{\sigma}\left|\frac{1}{\gamma_{j}}-\frac{1}{\lambda_{j}}\right||z| .
$$

Hence,

$$
\left|\operatorname{Im} \ln \frac{\Phi_{M}(z)}{\Psi_{M}(z)}\right| \leqslant \sum_{i \in M}|\arg | \frac{1-z / \lambda_{j}}{1-z / \gamma_{j}}|| \leqslant \frac{\pi}{\sigma} S_{p}|z|, \quad z \notin E_{M, \sigma} .
$$

The obtained estimates for $\operatorname{Re} \ln \frac{\Phi_{M}(z)}{\Psi_{M}(z)}$ and $\operatorname{Im} \ln \frac{\Phi_{M}(z)}{\Psi_{M}(z)}$ follow that

$$
\left|\ln \frac{\Phi_{M}(z)}{\Psi_{M}(z)}\right| \leqslant \frac{\sqrt{\pi^{2}+1}}{\sigma} S_{p}|z|, \quad z \notin E_{M, \sigma} .
$$


Outside set $E_{M, \sigma}$ the expression $\frac{\Phi_{M}(z)}{\Psi_{M}(z)}$ can be represented as $\exp c_{M}(z)$, where $c_{M}(z)=\ln \frac{\Phi_{M}(z)}{\Psi_{M}(z)}$. Expanding $\exp c_{M}(z)$ into the power series w.r.t. $c_{M}(z)$ and employing the standard way of estimating such series, we arrive at inequality (3.7).

3. We let $N=\{j>k, j \notin M\}$. Since $\{j: j>k\}=M \bigcup N$, function to be estimated can be represented as

$$
\psi_{k}(z) \varphi(z)-\varphi_{k}(z) \psi(z)=\psi_{k}(z) \varphi(z)\left(1-\frac{\Psi_{M}(z)}{\Phi_{M}(z)}\right)++\psi_{k}(z) \varphi(z) \frac{\Psi_{M}(z)}{\Phi_{M}(z)}\left(1-\frac{\Psi_{N}(z)}{\Phi_{N}(z)}\right)
$$

We note that set $M$ defined by formula $(3.9)$ is finite and this is why the set of indices $N$ differs from the set $\{j: j>k\}$ just by a finite number of elements. Thus, all four products involved in the definition of functions $\Phi_{M}, \Psi_{M}, \Phi_{N}, \Psi_{N}$ converge.

For fixed $\sigma \in(0 ; 1 / 2), R \geqslant \max \left\{2, \mu(\delta / \Delta)\left|\lambda_{k}\right|\right\}$ we choose a small positive number $\varepsilon_{R, \sigma}<2 \sigma R /(1-\sigma)$ so that the rectangle $\Pi_{R, \varepsilon}=\{z=x+\mathrm{i} y:|x| \leqslant R,|y| \leqslant \varepsilon\}$ and set $E_{N, \sigma}=\bigcup_{j \in N}\left(e_{\sigma}\left(\lambda_{j}\right) \bigcup e_{\sigma}\left(\gamma_{j}\right)\right)$ have no common internal points for each $\varepsilon \leqslant \varepsilon_{R, \sigma}$.

Let us estimate each of the terms in the right hand side of (3.11).

The expression $\varphi(z)\left(1-\frac{\Psi_{M}(z)}{\Phi_{M}(z)}\right)$ is an entire function. Employing condition 3.8), estimate (3.7) and restrictions for the choice of number $\varepsilon$, we obtain that on the boundary of the domain $G_{R, \varepsilon, \sigma}$ formed by all internal points of the set $\Pi_{R, \varepsilon} \cup\left(\bigcup_{j \in M}\left(e_{\sigma}\left(\lambda_{j}\right) \bigcup e_{\sigma}\left(\gamma_{j}\right)\right)\right)$ this entire function satisfies the inequality

$$
\begin{aligned}
\left|\varphi(z)\left(1-\frac{\Psi_{M}(z)}{\Phi_{M}(z)}\right)\right| \leqslant & \frac{\sqrt{\pi^{2}+1}(1+\sigma)}{\sigma(1-\sigma)} K_{M} S_{k+1} R \\
& \cdot \exp \left(\frac{\sqrt{\pi^{2}+1}(1+\sigma)}{\sigma(1-\sigma)} K_{M} S_{k+1}+\frac{\sigma\left(d_{\varphi}-c_{\varphi}\right)}{1-\sigma}\right) R .
\end{aligned}
$$

Taking into consideration relations (3.5), we obtain that as $R \geqslant \max \left\{2, \mu(\delta / \Delta)\left|\lambda_{k}\right|\right\}, k \in \mathbb{N}$ for each $x \in[-R ; R]$ the inequality

$$
\begin{aligned}
& \mid \psi_{k}(x) \varphi(x)\left(1-\frac{\Psi_{M}(x)}{\Phi_{M}(x)}\right) \mid \leqslant \frac{\sqrt{\pi^{2}+1}(1+\sigma)}{\sigma(1-\sigma)} K_{M} S_{k+1} R \\
& \cdot \exp \left[\left(\frac{\sqrt{\pi^{2}+1}(1+\sigma)}{\sigma(1-\sigma)} K_{M} S_{k+1}+\frac{\sigma\left(d_{\varphi}-c_{\varphi}\right)}{1-\sigma}\right) R+\min \{\delta R, k \ln R\}\right]
\end{aligned}
$$

holds true.

Let us estimate the second term in the right hand side of the representation (3.11). By inequalities (3.6), (3.8) and the choice of number $\varepsilon$, on the boundary of domain $G_{R, \varepsilon, \sigma}$ the entire function $\varphi(z) \frac{\Psi_{M}(z)}{\Phi_{M}(z)}$ satisfies the inequality

$$
\left|\varphi(z) \frac{\Psi_{M}(z)}{\Phi_{M}(z)}\right| \leqslant \exp \left(\frac{\sqrt{\pi^{2}+1}(1+\sigma)}{\sigma(1-\sigma)} K_{M} S_{k+1}+\frac{\sigma\left(d_{\varphi}-c_{\varphi}\right)}{1-\sigma}\right) R
$$

For each $\varepsilon \in\left(0 ; \varepsilon_{R, \sigma}\right)$ on the boundary of rectangle $\Pi_{R, \varepsilon}$ the term $\left(1-\frac{\Psi_{N}(z)}{\Phi_{N}(z)}\right)$ satisfies the estimate

$$
\left|\left(1-\frac{\Psi_{N}(z)}{\Phi_{N}(z)}\right)\right| \leqslant \frac{\sqrt{\pi^{2}+1}}{\sigma} S_{k+1}|z| \exp \left(\frac{\sqrt{\pi^{2}+1}}{\sigma} S_{k+1}|z|\right) .
$$


Inside rectangle $\Pi_{R, \varepsilon}$ function $\left(1-\frac{\Psi_{N}(z)}{\Phi_{N}(z)}\right)$ is analytic. Hence, for each $x \in[-R ; R]$,

$$
\left|\left(1-\frac{\Psi_{N}(x)}{\Phi_{N}(x)}\right)\right| \leqslant \frac{\sqrt{\pi^{2}+1}}{\sigma} S_{k+1} \sqrt{R^{2}+\varepsilon^{2}} \exp \left(\frac{\sqrt{\pi^{2}+1}}{\sigma} S_{k+1} \sqrt{R^{2}+\varepsilon^{2}}\right) .
$$

Passing to the limit as $\varepsilon \rightarrow 0$, we get

$$
\left|\left(1-\frac{\Psi_{N}(x)}{\Phi_{N}(x)}\right)\right| \leqslant \frac{\sqrt{\pi^{2}+1}}{\sigma} S_{k+1} R \exp \left(\frac{\sqrt{\pi^{2}+1}}{\sigma} S_{k+1} R\right) \quad x \in[-R ; R] .
$$

These inequality and estimates (3.14), (3.5) imply the required estimate for the second term:

$$
\begin{aligned}
\mid \psi_{k}(x) \varphi(x) & \frac{\Psi_{M}(x)}{\Phi_{M}(x)}\left(1-\frac{\Psi_{N}(x)}{\Phi_{N}(x)}\right) \mid \leqslant \frac{\sqrt{\pi^{2}+1}(1+\sigma)}{\sigma(1-\sigma)} K_{M} S_{k+1} R \\
& \cdot \exp \left[\left(\frac{2 \sqrt{\pi^{2}+1}(1+\sigma)}{\sigma(1-\sigma)} K_{M} S_{k+1}+\frac{\sigma\left(d_{\varphi}-c_{\varphi}\right)}{1-\sigma}\right) R+\min \{\delta R, k \ln R\}\right]
\end{aligned}
$$

for each $x \in[-R ; R], R \geqslant \max \left\{2, \mu(\delta / \Delta)\left|\lambda_{k}\right|\right\}, k \in \mathbb{N}$. Estimates (3.13) and (3.15) yield desired inequality 3.10 .

3.2. Stability conditions for submodule with two generators. We consider functions $\varphi, \psi \in \mathcal{F}\left(C_{0}^{\infty}(a ; b)\right) \subset \mathcal{P}$, obeying the conditions

$$
\varphi(0)=\psi(0)=1, \quad h_{\varphi}(\theta)=h_{\psi}(\theta), \quad \theta \in[0 ; 2 \pi) .
$$

Functions $\varphi$ and $\psi$ are Fourier-Laplace transforms of compactly supported infinitely differentiable functions and hence, they satisfy the estimates

$$
|\varphi(x)| \leqslant \frac{1}{|x|^{k}}, \quad|\psi(x)| \leqslant \frac{1}{|x|^{k}}, \quad x \in \mathbb{R}, \quad|x| \geqslant R_{k}, \quad k=1,2, \ldots,
$$

where $\left\{R_{k}\right\}$ is an increasing sequence of numbers greater than 2 .

We denote by $\Lambda=\left\{\lambda_{j}\right\}, \Gamma=\left\{\gamma_{j}\right\}$ the sequences of zeroes of functions $\varphi$ and $\psi$, respectively, taken in the ascending order of absolute values and each zero is written in accordance with its multiplicity. Sequences $\Lambda$ and $\Gamma$ have the same density; as above, we denote it by $\Delta_{0}$. For arbitrary fixed numbers $\Delta>\Delta_{0}, \delta>0$ we let $R_{j}^{*}=\mu(\delta / \Delta) \max \left\{\left|\lambda_{j}\right|,\left|\gamma_{j}\right|\right\}$, where function $\mu(\chi)$ is the inverse one for function $\chi(\mu)$ defined by formula 3.2 .

Theorem 1. Suppose that for some number $\Delta>\Delta_{0}, \delta>0$ and an increasing sequence $R_{k} \geqslant 2, k=1,2, \ldots$, satisfying (3.17) the identity

$$
\limsup _{k \rightarrow \infty} \frac{\ln \frac{1}{S_{k+1}}}{\max \left\{R_{k}, R_{k}^{*}\right\}}>\delta
$$

holds true. Then submodule $\mathcal{J}_{\varphi, \psi}$ generated by functions $\varphi$ and $\psi$ in module $\mathcal{P}$ is stable.

Proof. For a real number $c$ the mapping

$$
\varphi \mapsto \varphi_{c}=e^{\mathrm{i} c z} \varphi
$$

defines a topological isomorphism of original module $\mathcal{P}$ and module $\mathcal{P}_{c}$ formed by FourierLaplace transforms of distributions with compact supports lying in the interval $(a-c ; b-c)$. It is clear that submodule $\mathcal{J}_{\varphi, \psi}$ and its image $\mathcal{J}_{\varphi_{c}, \psi_{c}}$ under the mentioned isomorphism are stable or unstable simultaneously.

Making use of this fact, we pass to the functions $\varphi_{c}=e^{\mathrm{i} c z} \varphi, \psi_{c}=e^{\mathrm{i} c z} \varphi \in \mathcal{P}_{c}$, where $c=\left(h_{\varphi}(-\pi / 2)+h_{\varphi}(\pi / 2)\right) / 2$. The indicator diagrams of functions $\varphi_{c}$ and $\psi_{c}$ coincide with the segment $\left[-\mathrm{i} \pi \Delta_{0} ; \mathrm{i} \pi \Delta_{0}\right]$ of the imaginary axis. Hence, these functions satisfy estimate like (3.8). In what follows we omit subscript $c$. 
As it was mentioned above, module $\mathcal{P}$ is $b$-stable. Hence, in accordance with Proposition 4.2 in work [4] (in view of Remark 1 in Section 4 of the same work), it is sufficient to show the stability of closed submodule $\mathcal{J}$ for some point $\lambda_{0} \in \mathbb{C}$. For instance, for $\lambda_{0}=0$.

Let us employ the criterion of stability at point $\lambda_{0}$ for a submodule with a finite number of generators ([4, Prop. 4.9]). In the case of two generators it is formulated as follows: submodule $\mathcal{J}_{\varphi, \psi}$ with generators satisfying conditions $\varphi\left(\lambda_{0}\right)=1, \psi\left(\lambda_{0}\right)=1$ is stable at point $\lambda_{0}$ if and only if the identical zero can be approximated by functions $(p \varphi-q \psi)$ in the sense of topology of $\mathcal{P}$, where $p, q$ are polynomials and $p\left(\lambda_{0}\right)=q\left(\lambda_{0}\right)=1$.

In the beginning of Section 4 in work [4] containing Assumptions 4.5, 4.8 implying this criterion, on module $\mathcal{P}$ there imposed a stronger than $b$-stability condition on uniform stability. We recall that uniform stability of module $\mathcal{P}$ means that for each neighborhood $U \subset \mathcal{P}$ of zero there exists a neighborhood $U^{\prime} \subset \mathcal{P}$ of zero such that for each $\lambda \in \mathbb{C}$ the implication $\varphi \in U^{\prime}$, $n_{\varphi}(\lambda)>0 \Longrightarrow \frac{\varphi}{z-\lambda} \in U$ holds true. This notion was introduced in [3], 4].

In fact, as it was mentioned in Remark 2 in [4, Sect. 4], in the proof of Propositions 4.5, 4.8, 4.9 in [4] there was used just the following weaker property of pointwise stability of space $\mathcal{P}$ : for each neighborhood $U \subset \mathcal{P}$ of zero and each $\lambda \in \mathbb{C}$ there exists a neighborhood $V_{\lambda} \subset \mathcal{P}$ of zero such that $\varphi \in V_{\lambda}, n_{\varphi}(\lambda)>0 \Longrightarrow \frac{\varphi}{z-\lambda} \in U$. It was also proven in [4, Sect. 4] that a bornological $b$-stable space is pointwise stable.

By the said above we conclude that the formulated criterion of stability for a submodule with two generators can be applied for the considered module $\mathcal{P}$.

We note that the interesting for us properties of the submodule generated by functions $\varphi$ and $\psi$ are not influenced by changing a finite number of points in sequences $\Lambda$ and $\Gamma$. Indeed, suppose that for some $n_{0} \in \mathbb{N}$ for functions $\varphi / \varphi_{n_{0}}, \psi / \psi_{n_{0}}$ the stability criterion conditions hold true: there exist generalized sequence of polynomials $\tilde{p}_{\alpha}, \tilde{q}_{\alpha}$ obeying the identities

$$
\tilde{p}_{\alpha} \frac{\varphi}{\varphi_{n_{0}}}-\tilde{q}_{\alpha} \frac{\psi}{\psi_{n_{0}}} \rightarrow 0 \quad \text { in } \quad \mathcal{P}, \quad \tilde{p}_{\alpha}(0)=\tilde{q}_{\alpha}(0)=1 \quad \text { for each } \quad \alpha .
$$

Then the polynomials $p_{\alpha}=\varphi_{n_{0}} \psi_{n_{0}} \tilde{p}_{\alpha}, q_{\alpha}=\varphi_{n_{0}} \psi_{n_{0}} \tilde{q}_{\alpha}$ and functions $\varphi, \psi$ obviously satisfy the following relations:

$$
p_{\alpha} \varphi-q_{\alpha} \psi \rightarrow 0 \quad \text { in } \quad \mathcal{P}, \quad p_{\alpha}(0)=q_{\alpha}(0)=1 \quad \text { for each } \quad \alpha .
$$

Thus, we can assume that the original sequence of zeroes $\Lambda$ and $\Gamma$ satisfy conditions (3.3), (3.4).

We consider the sequence $\left\{\psi_{k} \varphi-\varphi_{k} \psi\right\}$. By (3.3) on real axis for $|x| \geqslant 2$ we have

$$
\left|\varphi_{k}(x)\right| \leqslant|x|^{k}, \quad\left|\psi_{k}(x)\right| \leqslant|x|^{k}, \quad k=1,2, \ldots
$$

Taking into consideration (3.17) and that $R_{k} \geqslant 2$, we get

$$
\left|\psi_{k}(x) \varphi(x)-\varphi_{k}(x) \psi(x)\right| \leqslant 2, \quad|x| \geqslant R_{k}, \quad k=1,2, \ldots
$$

We choose and fix number $\delta^{\prime}>\delta$ such that relation (3.18) remains true after the change of $\delta$ by $\delta^{\prime}$. There exists a subsequence of subscripts $k_{\nu}$ such that

$$
\frac{\ln \frac{1}{S_{k_{\nu}+1}}}{\max \left\{R_{k_{\nu}}, R_{k_{\nu}}^{*}\right\}}>\delta^{\prime}, \quad \nu=1,2, \ldots
$$

Hence,

$$
S_{k_{\nu}+1} \tilde{R}_{k_{\nu}} \rightarrow 0, \quad \nu \rightarrow \infty
$$

where we have indicated $\tilde{R}_{k}=\max \left\{R_{k}, R_{k}^{*}\right\}$.

We fix arbitrary $\sigma \in(0 ; 1 / 2)$ and make use of Item 3 in Lemma 2 letting $R=\tilde{R}_{k_{\nu}}$. First we estimate quantities $K_{M_{\nu}}$, where the set of subscripts $M_{\nu}=M\left(k_{\nu}, R_{k_{\nu}}, \sigma\right)$ is determined by 
formula 3.9. For $j \in M_{\nu}$ at least one of the quantities $\left|\lambda_{j}\right|,\left|\gamma_{j}\right|$ does not exceed $\tilde{R}_{k_{\nu}} /(1-\sigma)$. For instance, let $\left|\lambda_{j}\right| \leqslant \tilde{R}_{k_{\nu}} /(1-\sigma)$. Then

$$
|1-| \frac{\lambda_{j}}{\gamma_{j}}|| \leqslant S_{k_{\nu}+1} \tilde{R}_{k_{\nu}} /(1-\sigma) .
$$

Hence,

$$
K_{M_{\nu}} \rightarrow 1, \quad \nu \rightarrow \infty .
$$

By inequality (3.10) for the function $\left(\psi_{k_{\nu}} \varphi-\varphi_{k_{\nu}} \psi\right)$ we obtain the estimate

$$
\left|\psi_{k_{\nu}}(x) \varphi(x)-\varphi_{k_{\nu}}(x) \psi(x)\right| \leqslant \mathcal{M}_{1, \nu} \mathcal{M}_{2, \nu}, \quad|x| \leqslant \tilde{R}_{k_{\nu}}
$$

where

$$
\begin{aligned}
& \mathcal{M}_{1, \nu}=\frac{\sqrt{\pi^{2}+1}(1+\sigma)}{\sigma(1-\sigma)} K_{M_{\nu}}\left(1+\exp \left(\frac{\sqrt{\pi^{2}+1}(1+\sigma)}{\sigma(1-\sigma)} K_{M_{\nu}} S_{k_{\nu}+1} \tilde{R}_{k_{\nu}}\right)\right), \\
& \mathcal{M}_{2, \nu}=S_{k_{\nu}+1} \tilde{R}_{k_{\nu}} \exp \left[\left(\frac{\sqrt{\pi^{2}+1}(1+\sigma)}{\sigma(1-\sigma)} K_{M_{\nu}} S_{k_{\nu}+1}+\frac{2 \pi \Delta_{0} \sigma}{1-\sigma}\right) \tilde{R}_{k_{\nu}}+\delta \tilde{R}_{k_{\nu}}\right] .
\end{aligned}
$$

It follows from relations (3.21) and 3.22 that sequence $\left\{\mathcal{M}_{1, \nu}\right\}$ is bounded. For second term $\mathcal{M}_{2, \nu}$ we have

$$
\ln \mathcal{M}_{2, \nu}=\left[\left(\frac{\sqrt{\pi^{2}+1}(1+\sigma)}{\sigma(1-\sigma)} K_{M_{\nu}} S_{k_{\nu}+1}+\frac{2 \pi \Delta_{0} \sigma}{1-\sigma}+\frac{\ln \tilde{R}_{k_{\nu}}}{\tilde{R}_{k_{\nu}}}\right)+\delta-\frac{\ln \frac{1}{S_{k_{\nu}+1}}}{\tilde{R}_{k_{\nu}}}\right] \tilde{R}_{k_{\nu}} .
$$

We choose $\sigma$ close to zero so that the inequality $\left(2 \pi \Delta_{0} \sigma\right) /(1-\sigma)<\left(\delta^{\prime}-\delta\right) / 3$ holds true. For the chosen $\sigma$ we find the subscript $\nu=\nu_{\sigma}$ so that both the expressions $\frac{\sqrt{\pi^{2}+1}(1+\sigma)}{\sigma(1-\sigma)} K_{M_{\nu}} S_{k_{\nu}+1}$ and $\left(\ln \tilde{R}_{k_{\nu}}\right) /\left(\tilde{R}_{k_{\nu}}\right)$ are less than $\left(\delta^{\prime}-\delta\right) / 3$ for each $\nu \geqslant \nu_{\sigma}$.

Number $\delta^{\prime}$ and subsequence $\left\{k_{\nu}\right\}$ satisfy the relation

$$
\lim _{\nu \rightarrow \infty} \frac{-\ln S_{k_{\nu}+1}}{\max \left\{R_{k_{\nu}}, R_{k_{\nu}}^{*}\right\}}>\delta^{\prime} .
$$

This is why there exist positive number $\varepsilon_{0}$ and subscript $\nu=\nu_{1} \geqslant \nu_{\sigma}$ so that the expression in the square brackets in the right hand side of formula $(3.23)$ does not exceed $\left(-\varepsilon_{0}\right)$ for each $\nu \geqslant \nu_{1}$. Thus, we have the estimate

$$
\mathcal{M}_{2, \nu} \leqslant \exp \left(-\varepsilon_{0} \tilde{R}_{k_{\nu}}\right), \quad \nu \geqslant \nu_{1}
$$

Due to the boundedness of sequence $\left\{\mathcal{M}_{1, \nu}\right\}$ it yields that there exists an index $\nu_{0} \geqslant \nu_{1}$ such that

$$
\left|\psi_{k_{\nu}}(x) \varphi(x)-\varphi_{k_{\nu}}(x) \psi(x)\right| \leqslant 2, \quad|x| \leqslant \tilde{R}_{k_{\nu}}, \quad \nu \geqslant \nu_{0} .
$$

By these estimates and inequalities (3.19) we conclude that for each $\nu \geqslant \nu_{0}$ the inequalities

$$
\left|\psi_{k_{\nu}}(x) \varphi(x)-\varphi_{k_{\nu}}(x) \psi(x)\right| \leqslant 2, \quad x \in \mathbb{R}
$$

hold true. By Phragmén-Lindelöf principle the inequalities

$$
\left|\psi_{k_{\nu}}(z) \varphi(z)-\varphi_{k_{\nu}}(z) \psi(z)\right| \leqslant 2 \exp \left(\pi \Delta_{0}|\operatorname{Im} z|\right), \quad \nu \geqslant \nu_{0}
$$

are valid in the whole complex plane. These inequalities imply that the sequence of functions $\Phi_{\nu}(z)=\psi_{k_{\nu}}(z) \varphi(z)-\varphi_{k_{\nu}}(z) \psi(z), \nu=\nu_{1}, \nu_{2}, \ldots$, is bounded in $\mathcal{P}$ and thus, it is relatively compact in this space (cf. [1]). Thanks to the completeness of $\mathcal{P}$ (as a space of type $\left(L N^{*}\right)$ we conclude that some subsequence $\left\{\Phi_{\nu_{l}}\right\}$ converges to the identical zero in space $\mathcal{P}$.

In accordance with the aforementioned stability criterion in work [4], submodule $\mathcal{J}_{\varphi, \psi}$ is stable. 
Remark 1. Let $N_{0} \subset \mathbb{N}$ be an infinite set of indices so that for numbers $R_{k}$ the relation

$$
\lim _{k \rightarrow \infty, k \in N_{0}} \frac{R_{k}}{k}=+\infty
$$

holds true. Changing slightly the arguments in the latter part of the proof of Theorem 1 (concerning application of inequality (3.10), one can show that the theorem remains true if we replace condition (3.18) by the following one: there exists a subsequence $N_{1} \subset N_{0}$ satisfying

$$
\lim _{k \rightarrow \infty, k \in N_{1}} \frac{\ln \frac{1}{S_{k+1}}}{R_{k}}>0 .
$$

Corollary 1. Under the hypothesis of Theorem 1 the implication holds: if the set $\Lambda \bigcap \Gamma$ is finite, then submodule $\mathcal{J}_{\varphi, \psi}$ is weakly localizable.

Proof. Let $W$ be a $D$-invariant subspace in $\mathcal{E}$, for which $\mathcal{J}_{\varphi, \psi}$ is an annihilating submodule: $\mathcal{J}_{\varphi, \psi}=\mathcal{F}\left(W^{0}\right)$. In accordance with Theorem 1, submodule $\mathcal{J}_{\varphi, \psi}$ is stable and hence (Proposition 2), the spectrum of subspace $W$ is finite and is equal to $\left(-\mathrm{i} \Lambda_{\mathcal{J}_{\varphi, \psi}}\right)$. By Proposition 6.1 in work 12 subspace $W$ is the algebraic direct sum of subspaces $W_{I_{W}}$ and $\mathcal{L}\left(\operatorname{Exp}\left(-\mathrm{i} \Lambda_{\mathcal{J}}\right)\right)(\mathcal{L}(\cdot)$ stands for the linear span of a set). Employing the duality, we obtain that submodule $\mathcal{J}$ is the intersection of annihilating submodules of these spaces

$$
\mathcal{J}_{\varphi, \psi}=\mathcal{F}\left(W_{I_{W}}^{0}\right) \bigcap \mathcal{F}\left(\mathcal{L}\left(\operatorname{Exp}\left(-\mathrm{i} \Lambda_{\mathcal{J}}\right)\right)^{0}\right) .
$$

Submodule $\mathcal{F}\left(W_{I_{W}}^{0}\right)$ is the set of all functions in $\mathcal{P}$ whose indicator diagrams are contained in the set $\mathrm{i} I_{W}=\mathrm{i}\left[c_{\mathcal{J}} ; d_{\mathcal{J}}\right]$. Submodule $\mathcal{F}\left(\mathcal{L}\left(\operatorname{Exp}\left(-\mathrm{i} \Lambda_{\mathcal{J}}\right)\right)^{0}\right)$ is the set of all functions in $\mathcal{P}$ vanishing on set $\Lambda_{\mathcal{J}}$. Therefore, $\mathcal{J}_{\varphi, \psi}$ is a weakly localizable submodule.

\section{2-GENERATED SUBMODULES IN $\mathcal{P}$}

We apply the results of the previous section for proving the following theorem.

Theorem 2. Let $\mathcal{J} \subset \mathcal{P}$ be a stable submodule with a finite number of zeroes $\Lambda_{\mathcal{J}}$ and an indicator segment $\left[c_{\mathcal{J}} ; d_{\mathcal{J}}\right] \subset(a ; b)$, and ${ }^{1} c_{\mathcal{J}}<d_{\mathcal{J}}$. Then for each function $\varphi \in \mathcal{J}$ satisfying conditions $\varphi \in \mathcal{F}\left(C_{0}^{\infty}(a ; b)\right), h_{\varphi}(-\pi / 2)=c_{\mathcal{J}}, h_{\varphi}(\pi / 2)=d_{\mathcal{J}}$ there exists a function $\psi \in \mathcal{J}$ such that

$$
\mathcal{J}=\mathcal{J}_{\varphi, \psi}
$$

Proof. Without loss of generality we can assume that $0 \notin \Lambda_{\mathcal{J}}$.

Arguing as in the proof of Corollary 1, we conclude that submodule $\mathcal{J}$ is weakly localizable. Hence, the set $\mathcal{J} \cap \mathcal{F}\left(C_{0}^{\infty}(a ; b)\right)$ is non-empty. It is easy to see that among the functions of this set there exist function with the indicator diagram i $\left[c_{\mathcal{J}} ; d_{\mathcal{J}}\right]$. Let $\varphi \in \mathcal{J} \cap \mathcal{F}\left(C_{0}^{\infty}(a ; b)\right)$ be one of these function equalling 1 at point 0 and let $\Lambda$ be its zero set.

We choose and fix two numbers $a^{\prime}, b^{\prime} \in \mathbb{R}$ satisfying the inequalities

$$
a \leqslant a^{\prime}<c_{\mathcal{J}} \leqslant d_{\mathcal{J}}<b^{\prime} \leqslant b,
$$

and a sequence $\tilde{\Gamma}=\left\{\tilde{\gamma}_{k}\right\}$ close to $\Lambda$ so that sequences $\Lambda$ and $\tilde{\Gamma}$ satisfy (2.7). Let

$$
\tilde{C}=\sum_{j=1}^{\infty} \frac{\left|\lambda_{j}-\tilde{\gamma}_{j}\right|}{1+\left|\operatorname{Im} \lambda_{j}\right|}, \quad \tilde{A_{m}}=e^{2 \tilde{C}}\left\|s_{\varphi}^{(m+1)}\right\|_{L^{1}\left(a^{\prime} ; b^{\prime}\right)},
$$

where $s_{\varphi} \in C_{0}^{\infty}\left(a^{\prime} ; b^{\prime}\right)$ is the preimage under the Fourier-Laplace transform of function $\varphi$.

\footnotetext{
${ }^{1}$ If $c_{\mathcal{J}}=d_{\mathcal{J}}=c \in(a ; b)$, then submodule $\mathcal{J}$ is generated by one function $e^{-\mathrm{i} c z}$. It follows from Example 1 in [12, Sect. 2] mentioned in the beginning of Section 2 and the duality principle (Proposition 1).
} 
Let us consider an arbitrary sequence $\Gamma=\left\{\gamma_{k}\right\}, 0 \notin \Gamma$, so that

$$
\left|\gamma_{k}-\lambda_{k}\right| \leqslant\left|\tilde{\gamma}_{k}-\lambda_{k}\right|, \quad k=1,2, \ldots
$$

It follows from Proposition 3 and Lemma 1 that function $\psi$ determined by function $\varphi$ and sequence $\Gamma$ by identity 2.8 is the Fourier-Laplace transform of some function $s_{\psi} \in C_{0}^{\infty}\left(a^{\prime} ; b^{\prime}\right) \subset$ $C_{0}^{\infty}(a ; b)$, at that, ch $\operatorname{supp} s_{\psi}=\left[c_{\mathcal{J}} ; d_{\mathcal{J}}\right]$ and $\left|s_{\psi}^{(m)}(t)\right| \leqslant \tilde{A}_{m}, t \in(a ; b), m=0,1, \ldots$

Let $\left\{r_{k}\right\}_{k=0}^{\infty}$ be an increasing sequence of real numbers greater than 2 such that $|\varphi(x)| \leqslant|x|^{-k}$, $x \in \mathbb{R},|x| \geqslant r_{k}$. We let

$$
R_{k}=\max \left\{r_{k}, \tilde{A}_{k+1}\left(b^{\prime}-a^{\prime}\right)\right\}, \quad k=0,1,2, \ldots
$$

Function $\psi$ satisfies the relations

$$
|\psi(x)| \leqslant \frac{\tilde{A}_{k+1}\left(b^{\prime}-a^{\prime}\right)}{|x|^{k+1}} \leqslant \frac{1}{|x|^{k}}, \quad|x| \geqslant R_{k}, \quad k=0,1, \ldots
$$

These estimate hold true with the same $R_{k}$ for each function $\psi$ determined via formula (2.8) by function $\varphi$ and sequence $\Gamma$ once $\Gamma$ satisfies 4.1 . Among these sequences $\Gamma$ we choose that obeying additional conditions: the intersection $\Gamma \bigcap \Lambda$ is $\Lambda_{\mathcal{J}}$ and sequences $\Lambda$ and $\Gamma$ obey the hypothesis of Theorem 1 with numbers $R_{k}$ defined by formula 4.2 . Since $\mathcal{J}$ is a weakly localizable submodule, function $\psi$ determined by such sequence $\Gamma$ is contained in $\mathcal{J}$. By Theorem 1, submodule $\mathcal{J}_{\varphi, \psi}$ is stable and by Corollary 1 it is also weakly localizable.

Weakly localizable submodules $\mathcal{J}$ and $\mathcal{J}_{\varphi, \psi}$ have the same indicator segments and zero sets. Hence, $\mathcal{J}_{\varphi, \psi}=\mathcal{J}$.

Remark 2. Theorem 1 and the scheme of the proof of Theorem 2 can be employed for studying issue of 2-generatedness for stable submodules with an infinite zero set. We plan to discuss these issues in future publications.

The author expresses her gratitude to the participants of Ufa city seminar named after A.F. Leontiev on function theory for the attention to the work and useful discussion.

\section{BIBLIOGRAPHY}

1. J. Sebastian-e-Silva. "On some classes of locally convex spaces important in applications" // Matematika. Sbornik Perevodov. 1, 60-77 (1957). (in Russian).

2. B.Y. Levin (in collaboration with Yu. Lyubarskii, M. Sodin, V. Tkachenko). Lectures on entire functions. (Rev. Edition). AMS, Providence, Rhode Island (1996).

3. I.F. Krasičkov-Ternovskiı̌. Local description of closed ideals and submodules of analytic functions of one variable. I // Izvestia AN SSSR. Ser. Matem. 43:1, 44-66 (1979). [Math. USSR-Izvestiya. 14:1, 41-60 (1980).]

4. I.F. Krasičkov-Ternovskiı̌. Local description of closed ideals and submodules of analytic functions of one variable. II // Izvestia AN SSSR. Ser. Matem. 43:2, 309-341 (1979). [Math. USSRIzvestiya. 14:2, 289-316 (1980).]

5. I.F. Krasičkov-Ternovski1. Invariant subspaces of analytic functions. I. Spectral analysis on convex regions // Matem. Sbornik. 87(129):4, 459-489 (1972). [Math. USSR-Sbornik. 16:4, 471-500 (1972).]

6. N.F. Abuzyarova. A property of subspaces admitting spectral synthesis // Matem. Sbornik. 190:4, 3-22 (1999). [Sbornik: Math. 190:4, 481-499 (1999).]

7. N.F. Abuzyarova. Finitely generated submodules in the module of entire functions determined by restrictions on the indicator function // Matem. Zametki. 71:1, 3-17 (2002). [Math. Notes. 71:1, 3-16 (2002).]

8. B.N. Khabibullin. Spectral synthesis for the intersection of invariant subspaces of holomorphic functions // Matem. Sbornik. 196:3, 119-142 (2005). [Sbornik: Math. 163:3, 423-445 (2005).] 
9. B.N. Khabibullin. Closed submodules of holomorphic functions generated by submodules admitting local description // Trudy matem. centra im N.N. Lobachevskogo. Geometricheskaya teoriya funktsii i kraevye zadachi. 14, 280-298 (2002). (in Russian).

10. B.N. Khabibullin. Closed submodules of holomorphic functions with two generators // Funkts. Anal. Pril. 38:1, 65-80 (2004). [Funct. Anal. Appl. 38:1, 52-64 (2004).]

11. B.N. Khabibullin. Closed ideals of holomorphic functions with two generators // Matem. Zametki. 76:4, 604-609 (2004). [Math. Notes. 76:3-4, 558-563 (2004).]

12. A. Aleman, B. Korenblum. Derivation-Invariant Subspaces of $C^{\infty} / /$ Comput. Meth. Funct. Theory. 8:2, 493-512 (2008).

13. L. Hörmander. The analysis of linear partial differential operators I: distribution theory and Fourier analysis. Springer, Berlin, (1990).

14. A.M. Sedletskii. Analytic Fourier transforms and exponential approximations. I // Sovr. Matem. Fundament. Napravlenia. 5, 3-152 (2003). [J. Math. Sci. 129:6, 4251-4408 (2005).]

Natalia Fairbakhovna Abuzyarova,

Bashkir State University,

Z. Validi str., 32,

450074, Ufa, Russia

E-mail: abnatf@gmail.com 